\title{
Desarrollo de capacidades para el control del tabaco en América Latina
}

\author{
Mirta A Molinari,Abg, MD RRNN, (') María Lara Garrido-Herrero, Lic en D, MSc (') \\ Gustavo Sóñora-Parodi, Dr en D y CS. ${ }^{(1)}$
}

\section{Molinari MA, Garrido-Herrero ML, Sóñora-Parodi G. Desarrollo de capacidades para el control del tabaco en América Latina.} Salud Publica Mex 2010;52 supl 2:S298-S303.

\section{Resumen}

El Convenio Marco para el Control del Tabaco, primer tratado de salud pública del mundo aprobado en forma unánime por los 192 Estados miembros de la Organización Mundial de la Salud, constituye un hito en la promoción de la salud pública que incorpora una nueva dimensión jurídica en la cooperación internacional entre los países. La multidisciplinariedad de su temática y de los actores involucrados hacen necesaria la capacitación en áreas tales como gerencia, liderazgo, legislación, recursos humanos y financieros. Esto conduce a reforzar el desarrollo de la capacidad técnica y financiera existente en el ámbito nacional para que sus actividades sean exitosas y sostenibles en el tiempo, tanto desde el punto de vista de la voluntad política como de los recursos financieros.

Palabras clave: tabaquismo; educación; desarrollo sostenible
Molinari MA, Garrido-Herrero ML, Sóñora-Parodi G.

Development of capacities for tobacco

control in Latin America.

Salud Publica Mex 2010;52 suppl 2:S298-S303.

\section{Abstract}

The Framework Convention for Tobacco Control is the first global public health treaty. It was approved unanimously by the 192 Member States of the World Health Organisation. It is a landmark of public health promotion and it includes a new legal dimension for international cooperation between countries. The multidisciplinarity of the topics and the actors involved require building the capacity in areas such as: management, leadership, human and financial resources. This involves the reinforcement of the existing technical and financial capacity at national level, so the activities at that level can be successful and sustainable, gathering the political will and the financial resources needed for that.

Keywords: smoking; education; sustainable development apacity building es un término amplio considerado por varias disciplinas relacionadas especialmente con los temas ambientales y de desarrollo. Estudios y documentos internacionales lo han definido e interpretado como "desarrollo de capacidades", "creación de capacidad", "desarrollo de competencias", "aumento de la capacidad", entre otras. ${ }^{1}$
El desarrollo de capacidades abarca aspectos como el potencial humano, científico, tecnológico, de organización, institucional y del recurso económico del país. Uno de los objetivos fundamentales consiste en fortalecer la capacidad para evaluar y tratar las cuestiones de vital importancia relacionadas con las opciones políticas y los modos de implementar las alternativas de desarrollo,

(I) Unión Internacional Contra la Tuberculosis y Enfermedades Respiratorias, Departamento de Control de Tabaco, Oficina de México. México.

Fecha de recibido: 26 de marzo de 2010 • Fecha de aceptado: 21 de abril de 2010

Solicitud de sobretiros: Mirta A. Molinari, Oficina La Unión México.

Río Danubio 49, Col. Cuauhtémoc. 06500, México DF, México.

Correo electrónico:mmolinari@theunion.org, 
basados en la comprensión de los potenciales y de los límites del medio ambiente y las necesidades percibidas por la población del país en el que se aplican. En consecuencia, todos los países tienen la necesidad de fortalecer sus capacidades nacionales. ${ }^{2}$

En septiembre de 2000 tuvo lugar la Declaración del Milenio cuyos objetivos tienen fuerte sustento en una visión integral y amplia que apunta a la lucha contra la pobreza y el hambre, el deterioro ambiental, el mejoramiento de la educación y de la salud, entre otros. Quedó de manifiesto que dado que la falta de desarrollo es un problema que atañe y preocupa a todo el mundo y no solamente a los países menos desarrollados, el establecimiento de una alianza que enriquezca y revitalice la cooperación internacional, haciéndola más adecuada y efectiva, debía de ser uno de los objetivos seleccionados. ${ }^{3}$

La Declaración de Doha, por su parte, reconoce las necesidades de los países en desarrollo y menos adelantados de potenciar el apoyo a la asistencia técnica y la creación de capacidad capacity building en esta esfera, incluidos el análisis y la formulación de políticas que puedan evaluar mejor las consecuencias de una cooperación multilateral más estrecha para sus políticas y objetivos de desarrollo, tanto institucional como humano. ${ }^{4}$

El Convenio Marco para el Control del Tabaco $(\mathrm{CMCT})^{5}$ en su preámbulo reconoce que el control del tabaco en todos los niveles, particularmente en los países en desarrollo y en los países con economías en transición, necesita de recursos financieros y técnicos suficientemente adecuados a las necesidades actuales y especialmente previstos para esa actividad. ${ }^{5}$

Asimismo, expresa que las dificultades sociales y económicas que pueden generar a mediano y largo plazo los programas de control de tabaco en algunos países en desarrollo o con economías en transición requieren de asistencia técnica y financiera en el contexto de las estrategias de desarrollo sostenible formuladas a nivel nacional. ${ }^{5}$

La Organización de Naciones Unidas para el Desarrollo (UNDP) explica que el desarrollo de capacidades es un proceso continuo, de largo plazo, en el que deben participar todos los interesados, entendiéndose por tales los gobiernos nacionales y estatales, las organizaciones de la sociedad civil, las asociaciones profesionales y académicas, así como otros actores. ${ }^{6}$

La globalización de la economía y el intrincado escenario que la compone, tanto en su temática como en los actores que se encuentran involucrados, obliga a éstos a capacitarse no solamente en los temas específicos sino a ampliar su alcance a ciertas áreas que hace algunos años hubiesen parecido no pertener al control de tabaco, tales como gerencia, liderazgo, legislación, recursos humanos y financieros. Estos conceptos nos llevan a reforzar las capacidades existentes a nivel nacional para que la implementación de sus actividades sea exitosa y sostenible en el tiempo, tanto desde el punto de vista de la voluntad política como de los recursos financieros.

El desarrollo de capacidad técnica y financiera comprende:

- El desarrollo de recursos humanos, que incluye el proceso de equipar a individuos en la comprensión, habilidades y acceso a la información, al conocimiento y al entrenamiento que les permita desempeñarse con eficacia.

- El desarrollo de una organización, la elaboración de las estructuras de gerencia, procesos y procedimientos, no sólo dentro de las organizaciones sino también de la gerencia de relaciones entre las diversas organizaciones y sectores (público, privado y comunidad).

- El desarrollo del marco institucional y jurídico que conllevan los cambios legales y regulatorios para permitir a las organizaciones, a las instituciones y a las agencias en todos los niveles y en todos los sectores realizar sus capacidades.

- El desarrollo de capacidad técnica y financiera también se centra en una serie de acciones dirigidas a los participantes para ayudarlos en el incremento de sus conocimientos, habilidades y entendimiento, y que les permite desarrollar las aptitudes necesarias para producir el cambio deseado, con una perspectiva de largo plazo y una visión de cambio social. Es un proceso endógeno que debe realizarse teniendo en cuenta el contexto en el cual se lleva a cabo y es importante que involucre diferentes sectores y niveles. Requiere de liderazgo y decisión política al ser un proceso de largo alcance que se obtiene por medio del compromiso y la perseverancia. ${ }^{7}$

El CMCT instaló definitivamente en la comunidad internacional el control de tabaco como uno de los temas prioritarios en las agendas de salud. El éxito obtenido, sin embargo, se convierte indiscutiblemente en un desafío para los Estados parte, quienes deberán implementarlo en el ámbito nacional, regional y global como un medio para reducir el consumo y mitigar los efectos que produce en la salud, sociedad, medio ambiente y economía.

Consciente de estas necesidades, la Unión Internacional Contra la Tuberculosis y Enfermedades Respiratorias (La Unión) ha desarrollado e implementado varias herramientas para asistir a los países en el desarrollo de 
sus capacidades por medio de programas de desarrollo gerencial y de capacitación jurídica.

\section{Programa Internacional de Desarrollo Gerencial de la Unión}

La Unión ha diseñado una serie de cursos para apoyar a los países en la mejora de la gestión de sus programas de salud. ${ }^{8}$ El programa que engloba estos cursos se denomina Programa Internacional de Desarrollo Gerencial.

Los cursos inicialmente se enfocaban en los desafíos que encontraban los gestores de programas de salud nacionales y administradores de servicios de salud que disponían de recursos limitados. Las áreas en las que se impartían eran el control de la tuberculosis y la gestión de servicios de salud.

En 2006 se establece la Iniciativa Bloomberg 9 que pretende revertir la epidemia global del tabaco a partir de cuatro intervenciones clave: aplicar impuestos para aumentar el precio e impedir el contrabando; cambiar la imagen del tabaco al prohibir la publicidad directa e indirecta y organizar campañas de educación pública de alto impacto para el control del tabaco; proteger a la población contra la exposición al humo de tabaco; y ayudar a los fumadores a dejar el tabaco.

Por medio de estos recursos se apoyan iniciativas del sector público de países de medianos y bajos ingresos para implementar las intervenciones clave, educar a las comunidades e impulsar los cambios de políticas y monitorear rigurosamente el estatus global del consumo del tabaco y el progreso de países en la implementación de las medidas de control del tabaco. Con este objetivo se decidió adaptar los cursos al área del control del tabaco para un nuevo público: personas con nivel gerencial medio y alto cuyas responsabilidades son la implementación de estos programas en los aspectos administrativos. Los beneficiarios pueden pertenecer a organizaciones no gubernamentales nacionales, regionales o internacionales, así como a órganos gubernamentales.

Los cursos tienen como objetivo desarrollar las capacidades esenciales que se requieren para gestionar programas de control del tabaco como, por ejemplo, gestión de proyecto, presupuesto y administración, desarrollo de técnicas de liderazgo exitosas y gestión de recursos humanos. Cada curso es parte de un proceso educacional que sigue una progresión natural para la capacitación del gestor.

La capacitación ofrece experiencias de aprendizaje prácticas e intensivas; los participantes realizan evaluaciones, estudios de caso, simulaciones, ejercicios e intercambio de experiencias. El objetivo principal es capacitar a los participantes para que contribuyan personalmente a hacer una diferencia en el control del tabaco en sus países. El Programa aprovecha los conocimientos de los participantes y promueve el intercambio de experiencias e información en su ámbito de trabajo. Incluye la elaboración de planes de acción individuales así como la realización de ejercicios de coaching, los cuales tienen un impacto inmediato y directo.

Los participantes se seleccionan con los siguientes criterios: organizaciones que estén llevando a cabo proyectos en países prioritarios financiados por la Iniciativa (México y Brasil para América Latina) y organizaciones que hayan presentado ideas de proyecto o propuestas completas, pero que no hayan alcanzado exitosamente el proceso de selección. Se invita a una persona por organización pues la idea es que, al capacitar a una persona, ésta pueda retransmitir los conocimientos a sus compañeros. Asimismo, se requiere que al menos participen dos personas por país, con un balance entre representantes de la sociedad civil y de gobiernos.

A marzo del 2010, La Unión ha impartido ya dos ciclos completos de cursos del Programa en América Latina y está en el proceso de impartir su tercer ciclo.

Los resultados de las evaluaciones de los dos primeros ciclos demostraron que los participantes se identifican más con los facilitadores de su misma región, que cuentan con experiencias cercanas y con los materiales en su propio idioma.

Para mejorar el Programa se llevan a cabo evaluaciones de distintos tipos: de conocimiento, que se realiza en forma diaria durante el curso, y de nivel de satisfacción de los participantes, que se efectúa al final del curso por medio de un cuestionario. La evaluación del aprovechamiento se realiza tres meses después de haber finalizado el último curso, dándole seguimiento al plan de acción que cada participante creó durante el primer curso y que incluye consideraciones como el empleo de los conocimientos adquiridos durante el curso en el ejercicio de las tareas de su puesto una vez de regreso en la organización de origen.

Además de estos indicadores se utiliza un indicador adicional. Tras la participación en un ciclo de cursos se observa el número de ideas de proyecto presentadas para la Iniciativa Bloomberg y la calidad de éstas, así como la calidad de los informes presentados por las organizaciones que reciben fondos para llevar a cabo sus proyectos.

Finalmente, se han observado beneficios adicionales de la participación en los cursos del Programa Internacional de Desarrollo Gerencial. En primer lugar, el equipo de la Unión ha tenido oportunidad de entrar en contacto con organizaciones que trabajan en el control del tabaco en la región de América Latina y definir mejor las necesidades técnicas que existen en la región. 
Además, los participantes han expresado la utilidad de estos cursos para reforzar, crear y mantener relaciones con organizaciones de otros países, lo cual facilita el intercambio de información durante y después de los cursos, e impacta positivamente en la creación de un movimiento regional para el control del tabaco.

\section{Capacitación en el área jurídica}

El CMCT expresa que las vías administrativas, ejecutivas o legislativas (artículos 4 y 8), ${ }^{*, 5}$ u otras medidas eficaces son las adecuadas para su implementación.

El Estado actualiza su obligación ${ }^{10}$ de proteger la salud de la población en cumplimiento del Tratado a partir de la promulgación de normas jurídicas.

Los acuerdos voluntarios que los Estados realicen con grupos comerciales de interés o las ofertas de éstos para su autorregulación son contrarios al texto del Tratado e ineficaces, pues retrasan el avance hacia la adecuada protección a la salud de los habitantes de un país. ${ }^{11}$

Nuestra región cuenta con excelentes recursos técnicos a nivel de los poderes Ejecutivo, Legislativo y Judicial, así como en las organizaciones de la sociedad civil en cuanto a su acción en la protección del derecho a la salud.

La recopilación y difusión de evidencia científica y jurídica conducen a fortalecer las capacidades preexistentes.

El control de tabaco como especie dentro del género del derecho a la salud contiene particularidades como, por ejemplo, la ausencia -hasta tiempo reciente- de normas jurídicas de implementación del CMCT que permitan el análisis en el ámbito del derecho comparado; aprobación progresiva de directrices (o protocolos) por la conferencia de las partes, que deben ser interpretadas e incorporadas en posteriores ordenamientos jurídicos nacionales y la acción promo-

\footnotetext{
* Art. 4, Principios básicos: Para alcanzar los objetivos del Convenio y de sus protocolos y aplicar sus disposiciones, las Partes se guiarán, entre otros, por los principios siguientes: 1. [...] se deben contemplar en el nivel gubernamental apropiado medidas legislativas, ejecutivas, administrativas $\mathrm{u}$ otras medidas para proteger a todas las personas del humo de tabaco. [...]

Art. 8, Protección contra la exposición al humo de tabaco: ... 2. Cada Parte adoptará y aplicará, en áreas de la jurisdicción nacional existente y conforme determine la legislación nacional, medidas legislativas, ejecutivas, administrativas $\mathrm{y} / \mathrm{u}$ otras medidas eficaces de protección contra la exposición al humo de tabaco en lugares de trabajo interiores, medios de transporte público, lugares públicos cerrados y, según proceda, otros lugares públicos, y promoverá activamente la adopción y aplicación de esas medidas en otros niveles jurisdiccionales.
}

vida por grupos de interés innovando estrategias con la finalidad de postergar e impedir el avance jurídico en la implementación del CMCT. ${ }^{*, \neq}, 5$

La capacitación jurídica es una actividad dinámica y prioritaria que puede visualizarse al menos desde dos ángulos: el de las organizaciones internacionales que trabajan en la promoción de la salud -implementación y consolidación del CMCT-, y que tendrán el deber de ofrecerla con máxima calidad, y desde los sectores gubernamental y no gubernamental que promueven la protección de la salud, y que deben tomarla como una etapa de su formación e internalizar al máximo la evidencia científica y jurídica para, a posteriori, provocar el desdoble en su adaptación a su contexto sociopolítico.

La tarea de capacitación se dirige a los sectores gubernamentales y no gubernamentales porque del esfuerzo integral se deriva el éxito.

Los participantes de la sociedad civil son seleccionados principalmente, aunque no excluyentemente, por su trabajo en organizaciones relacionadas con los derechos humanos y el derecho a la salud. En el sector gubernamental se dirige a integrantes de los poderes del Estado.

Posteriormente, a partir de variados indicadores como referencias, actividades previas, etc., además de un cuestionario, se elabora un perfil del grupo para alcanzar una capacitación uniforme, además de que son consideradas muy especialmente sus opiniones a efectos de proyectar necesidades, oportunidades y futuras actividades.

\footnotetext{
* Todo derecho, entonces, requiere para su efectividad obligaciones positivas y negativas. En línea con esta idea, autores como Fried van Hoof o Asbjørn Eide proponen un esquema interpretativo consistente en el señalamiento de "niveles" de obligaciones estatales, que caracterizarían el complejo que identifica a cada derecho, independientemente de su adscripción al conjunto de derechos civiles o al de derechos sociales. De acuerdo a la propuesta de van Hoof, por ejemplo, podrían discernirse cuatro "niveles" de obligaciones: obligaciones de respetar, obligaciones de proteger, obligaciones de asegurar y obligaciones de promover el derecho en cuestión. Las obligaciones de respetar se definen por el deber del Estado de no injerir, obstaculizar o impedir el acceso al goce de los bienes que constituyen el objeto del derecho. Las obligaciones de proteger consisten en impedir que terceros interfieran, obstaculicen o impidan el acceso a esos bienes. Las obligaciones de asegurar suponen asegurar que el titular del derecho acceda al bien cuando no puede hacerlo por sí mismo. Las obligaciones de promover se caracterizan por el deber de desarrollar condiciones para que los titulares del derecho accedan al bien.

₹ El inciso 3 del artículo 5 del CMCT señala: A la hora de establecer y aplicar sus políticas de salud pública relativas al control del tabaco, las Partes actuarán de una manera que proteja dichas políticas contra los intereses comerciales y otros intereses creados de la industria tabacalera, de conformidad con la legislación nacional.
} 
Recientemente, como consecuencia de la vigencia de normas jurídicas ha surgido un hecho nuevo: los recursos judiciales o administrativos que interponen grupos de interés que consideran afectados sus derechos por las regulaciones jurídicas para obtener la inaplicabilidad de la regulación. Esto genera un desafío en el alcance y en la forma de la capacitación dirigida a nuevos actores que serán los protagonistas en la consolidación de los procesos jurídicos en curso, para no retroceder en el avance alcanzado en la protección de la salud, como por ejemplo: poder Judicial y departamentos contenciosos de los Ministerios/Secretarías de Salud / Fiscalías.

Es necesario que los sectores involucrados comprendan el por qué de las medidas jurídicas contenidas en el CMCT a efecto de que su convicción personal coadyuve en la implementación de las regulaciones y que la ausencia de información no derive en una inconsciente actitud de morigerar el alcance los objetivos.

Detrás de cada una de las medidas que el CMCT demanda implementar, existe el respaldo de evidencia científica.

Desde la vigencia del CMCT, los principios jurídicos inherentes al control de tabaco deben conectarse al contexto jurídico del derecho a la salud, como especie dentro del género. Por lo tanto, desde el punto de vista jurídico se hace énfasis en:

a) Disponer de una base jurídica amplia en cuanto a protección del derecho a la salud y por consiguiente, conocer cuál es la situación jurídica de un Estado ante la ratificación del $\mathrm{CMCT},{ }^{*, 3,11}$ como cimiento de la futura acción.

ElCMCT habrá de interpretarse conjuntamente con el derecho positivo nacional que consagra el derecho a la salud: la Constitución de la República y otros tratados internacionales, como por ejemplo, Pacto Internacional de Derechos Económicos, Sociales y Culturales ${ }^{12}$ y Convención sobre los Derechos del Niño. ${ }^{13}$

b) La legislación comparada tiene su importancia en que de su análisis crítico emerge una herramienta

\footnotetext{
* Art. 4, Principios Básicos: Para alcanzar los objetivos del Convenio y de sus protocolos y aplicar sus disposiciones, las Partes se guiarán, entre otros, por los principios siguientes:

1. Todos deben estar informados de las consecuencias sanitarias, la naturaleza adictiva y la amenaza mortal del consumo de tabaco y de la exposición al humo de tabaco y se deben contemplar en el nivel gubernamental apropiado medidas legislativas, ejecutivas, administrativas u otras medidas para proteger a todas las personas del humo de tabaco. [...]
}

valiosa para enriquecer antecedentes y adecuarlos a las realidades nacionales.

c) Los argumentos jurídicos y estrategias de los sectores de interés involucrados (como por ejemplo la industria tabacalera) que persiguen el retraso en la implementación del CMCT son comunes y, como consecuencia, son sistematizados. Su difusión y conocimiento por los Estados permitirán una actuación anticipada a los argumentos que inevitablemente esos sectores esgrimirán y, como resultado, será más eficaz la instrumentación de políticas públicas en salud.

La conexión e intercambio entre profesionales de derecho de países de la región será una herramienta, hasta hoy incipiente, pero que tal vez en el futuro inmediato tendrá un valor cualitativo trascendente.

d) Es necesario que se conozcan las vías de implementación del CMCT, ya sean administrativas, ejecutivas o legislativas, para actuar en consecuencia y adecuarlas de la mejor forma a la realidad de un país.

Actualmente, el poder Judicial comienza a ser protagonista en la consolidación del proceso de control de tabaco, y con esto se habilita una cuarta vía de acción, la "vía judicial".

En efecto, la hipótesis de que los poderes políticos del Estado no implementan o implementan inadecuadamente el CMCT en la protección del derecho a la salud permite el tránsito hacia este camino "judicial", ${ }^{10}$ previsto por el CMCT cuando se refiere a "otras medidas eficaces" como vías alternativas a las administrativas, ejecutivas o legislativas.

\section{Conclusión}

¿Por qué adquieren relevancia las acciones de capacitación que nuestras organizaciones desarrollan en la región?

El desarrollo de capacidades técnicas, financieras y en recursos humanos es esencial. Sin la educación y entrenamiento de los recursos humanos la mayoría de las intervenciones serían ineficaces.

Las actividades regionales de capacitación dejan excelentes aprendizajes ya que el intercambio de experiencias es la génesis de algo todavía más ambicioso: la generación de una política supranacional por bloques o coaliciones regionales.

El objetivo es que los sectores involucrados sean eficientes en el empleo de sus capacidades y, para ello, 
el conocimiento de todas las herramientas disponibles es el nexo para la obtención del objetivo buscado.

Una norma jurídica sólida y fundamentada es la mejor garantía en la protección de la salud de la población de un país, pero, asimismo, lo es para que se puedan sortear con éxito las acciones o recursos que se interpongan para su revocación o inaplicación.

Es imperioso continuar trabajando e incrementar los logros obtenidos hasta el presente para revertir la pandemia tabáquica por medio de intervenciones clave de probada costo-efectividad para gestionar los programas de salud.

\section{Declaración de conflicto de intereses}

Declaramos no tener conflicto de intereses.

\section{Referencias}

I. Organización Mundial de la Salud. Convenio Marco para el Control del Tabaco. Ginebra: Suiza, 2003.

2. Charles Lusthaus, Marie-Hélène Adrien, Mark Perstinger. Capacity Development: Definitions, Issues and Implications for Planning, Monitoring and Evaluation Universalia Ocassional Paper, №35, Canada, Septiembre, 1999
3. The United Nations Conference on Environment and Development. Agenda 2I, National mechanisms and international cooperation for capacity-building, Rio de Janeiro, Naciones Unidas, 1992: 37.1 - 37.13 4. Asamblea General de las Naciones Unidas. Declaración del Milenio. Naciones Unidas. Nueva York, 2000.

5. Conferencia Mundial de la Organización Mundial del Comercio, Declaración Ministerial de Doha, Qatar, Organización Mundial del Comercio, 200I.

6. UNDP. Human Development Reports. Oxford University Press, New York, 1991, 1997, I998

7. World Health Organization. Building Blocks for tobacco control:A handbook. Geneve. Suiza, 2004.

8. Programa Internacional de Desarrollo Gerencial de la Unión Internacional Contra la Tuberculosis y Enfermedades Respiratorias, Paris: International Union Against Tuberculosis and Lung Disease, [consultado 2010 abr 16] Disponible en: http://www.union-imdp.org/content. asp? pageid $=8 \&$ topid $=1$

9. Bloomberg Initiative to Reduce Tobacco Use Grants Programme; 2009 [actualizado 2009; consultado $2010 \mathrm{abr}$ 16]. Disponible en: http://www. tobaccocontrolgrants.org/Pages/44/About-the-Bloomberg-Initiative 10. Abramovich V, Courtis C. Apuntes sobre la exigibilidad judicial de los derechos sociales. Jura Gentium Revista de filosofía del derecho internacional y de la política global, I Año 2005 [consultado $2010 \mathrm{abr}$ |6]. Disponible en http://www.juragentium.unifi.it/es/surveys/latina/courtis.htm II. Madrazo-Lajous A, Zambrano-Porras JL. Análisis jurídico del convenio entre la SSA y la industria tabacalera. Estudio de caso, Ensayo, Salud Pública Méx 2007; Vol. 49(2):290-30I

12. Asamblea de las Naciones Unidas, 16 de Diciembre de 1966 y Preámbulo del Convenio Marco para el Control del Tabaco. Ginebra: Suiza, 2003.

13. Asamblea de las Naciones Unidas, 20 de Noviembre de 1989. 